\title{
Gender and Rapid Alterations of Hemispheric Dominance during Planning
}

\author{
Daniel Schuepbach ${ }^{\mathrm{a}}$ Tatjana Skotchko $^{\mathrm{a}}$ Stefan Duschek ${ }^{\mathrm{c}}$ \\ Anastasia Theodoridou $^{b}$ Simone Grimm ${ }^{a}$ Heinz Boeker ${ }^{a}$ Erich Seifritz ${ }^{a}$ \\ ${ }^{a}$ Clinic for Affective Disorders and General Psychiatry and ${ }^{b}$ Clinic for General and Social Psychiatry, \\ Psychiatric University Hospital Zurich, Zurich, Switzerland; ' Department of Psychology, University of Munich, \\ Munich, Germany
}

\section{Key Words}

Cerebral hemodynamics - Functional transcranial Doppler •

Gender $\cdot$ Laterality $\cdot$ Planning

\begin{abstract}
Background: Mental planning and carrying out a plan provoke specific cerebral hemodynamic responses. Gender aspects of hemispheric laterality using rapid cerebral hemodynamics have not been reported. Method: Here, we applied functional transcranial Doppler sonography to examine lateralization of cerebral hemodynamics of the middle cerebral arteries of 28 subjects ( 14 women and 14 men) performing a standard planning task. There were easy and difficult problems, and mental planning without motor activity was separated from movement execution. Results: Difficult mental planning elicited lateralization to the right hemisphere after 2 or more seconds, a feature that was not observed during movement execution. In females, there was a dominance to the left hemisphere during movement execution. Optimized problem solving yielded an increased laterality change to the right during mental planning. Conclusions: Gender-related hemispheric dominance appears to be condition-dependent, and change of laterality to the right may play a role in optimized performance. Results are of relevance when
\end{abstract}

considering laterality from a perspective of performance enhancement of higher cognitive functions, and also of psychiatric disorders with cognitive dysfunctions and abnormal lateralization patterns such as schizophrenia.

Copyright $\odot 2012$ S. Karger AG, Basel

\section{Introduction}

Routine and nonroutine planning are essential cognitive operations for normal levels of functioning. The temporal integration of goal-directed behavior [1] is a cornerstone of normal prefrontal functioning. Ample evidence exists that patients with lateral prefrontal lesions are severely disrupted in planning ability [2]. The lateral part of the prefrontal cortex, especially the dorsolateral prefrontal cortex (DLPFC), is an essential neuroanatomical component during planning [1]. The activation of neural circuits typically involves the DLPFC, the cingulate cortex, basal ganglia, the thalamus and the cerebellum [3]. Females rely on cerebral regions thought to be involved

This research was presented at the 8th FENS Forum, July 14-18, 2012, in Barcelona, Spain.

\section{KARGER}

Fax +41613061234 E-Mail karger@karger.ch www.karger.com
(C) 2012 S. Karger AG, Basel

0302-282X/12/0663-0149\$38.00/0

Accessible online at:

www.karger.com/nps
Daniel Schuepbach, MD

Clinic for Affective Disorders and General Psychiatry

Psychiatric University Hospital Zurich, Lenggstrasse 31, PO Box 1931

$\mathrm{CH}-8032$ Zurich (Switzerland)

Tel. +41 4438421 11, E-Mail daniel.schuepbach@ @uk.zh.ch 
in executive processing, e.g. the DLPFC, and males use regions specialized in visuospatial processing such as the posterior parietal cortex [4]. However, the current neuroimaging literature on planning and gender has not been presented information on the temporal 'Gestalt' of laterality during separate phases of standardized planning tasks, especially with respect to gender.

Hemispheric dominance has been relevant for paradigms that tap visuospatial or verbal abilities, with a right or left hemispheric activation pattern, respectively. Males, for example, show better performance in visuospatial abilities whereas females solve tasks of verbal and language domain more efficiently. Evidence suggests that there is distinct brain activity between males and females during mental rotation, verbal and visuospatial tasks [5, 6]. This is also in line with evidence that males and females use diverse activation patterns during a variety of cognitive tasks [7], even though there may be no performance difference in some.

An elegant technique for the measurement of rapid changes of cerebral blood flow and hemispheric laterality is functional transcranial Doppler sonography (fTCD). It allows the registration of intracranial blood flow parameters under defined conditions of stimulation in a noninvasive and uncomplicated way [8]. Changes in cerebral blood flow velocity (MFV) of basal cerebral arteries reflect changes in the blood demand of the respective arterial territories and hence respective neural activity. A number of studies have shown that fTCD is a suitable technique for the detection of minute MFV changes due to neuropsychological stimuli $[9,10]$. Frauenfelder et al. [11] were able to show a characteristic MFV increase during the Stockings of Cambridge (SOC), a standardized planning task that on the one hand differed from a visuomotor control task and on the other hand depended on the level of problem difficulty. Complex cognitive functions such as nonroutine planning do not provoke a clear hemispheric pattern, nor is there evidence of solid performance discrepancies between both genders. For example, Misteli et al. [12] were able to show subtle changes of hemispheric dominance between genders due to such a task, alterations that have not been described before. It is, therefore, interesting to investigate laterality and its dynamics during planning, be it for a mixed sample or gender-wise.

This study addressed the following questions: first, how does routine and nonroutine planning affect hemispheric dominance during two separate phases of mental planning and movement execution? Second, are there gender differences of hemispheric dominance? Third, is there a link between hemispheric dominance and task performance, especially when considering fast changes of laterality?

\section{Methods}

Subjects and Tasks

Twenty-eight healthy volunteers (14 men and 14 women, mean age $31.5 \pm 7.6$ years) participated in this study. The subjects were right-handed as assessed by the Salmaso and Longoni handedness inventory [13]. The local ethical committee approved the study and subjects gave written informed consent. They were not allowed to consume caffeine or nicotine for $2 \mathrm{~h}$ prior to the experiment.

\section{Technical Settings}

The fTCD measurements were carried out with a Multi-Dop X instrument (DWL Elektronische Systeme GmbH, Sipplingen, Germany). Two dual 2-MHz transducers were attached and fixed with a headband. Both middle cerebral arteries (MCA) were insonated at depths of $48-55 \mathrm{~mm}$ through the temporal bone window using standard diagnostic procedures [14] and making use of the continuous M-Mode of the apparatus allowing the identification of direction and maximum flow [15]. Peak MFV was simultaneously assessed in both examined vessels. The SOC was conducted with a personal computer (Compaq Computer Inc., Houston, Tex., USA), which was connected to a touch-screen monitor. A second monitor was positioned beside the test screen to show a standard screensaver program. During rest phases, individuals looked at the computer screen, which was running a conventional screen saver (Starfield; Microsoft Corp., Redmond, Wash., USA) [11]. Patients and subjects were told to sit in an upright and comfortable position and to breathe normally. Additional parameters like $\mathrm{pCO}_{2}$ and blood pressure were not registered, since there is evidence that these covariates, even when moderately changing, do not appreciably alternate the vessel diameter of the MCA [16]. Subjects were asked to refrain from talking and unnecessary movements.

\section{Cognitive Task}

The SOC has been described elsewhere in detail $[17,18]$. Briefly, the SOC was displayed on a touch-screen monitor that was divided into an upper part (goal state) and a lower part (start state), each containing three pockets (stockings) of different sizes. Three differently colored balls were located in the goal and the start configuration. As the goal and the start configuration varied the subject's task consisted of copying the goal configuration by moving the balls in the start configuration. Subjects moved the balls by selecting them with their right index finger. Hence, there were two SOC phases: first, mental planning (planning without moving) and second, movement execution (carrying out planned movements) (fig. 1). After a certain number of completed problems a control task was inserted, which required subjects to copy one move at a time in exact correspondence with the moves made in the preceding planning condition. The control task matched the previous planning problems in terms of the number of moves and comprised the same number of trials (i.e. 8 planning tasks and 8 control tasks). Hence, there was a control for the motor and visual component of the SOC. 
Fig. 1. Schematic illustration of SOC phases: mental planning, movement execution and visuomotor control task.

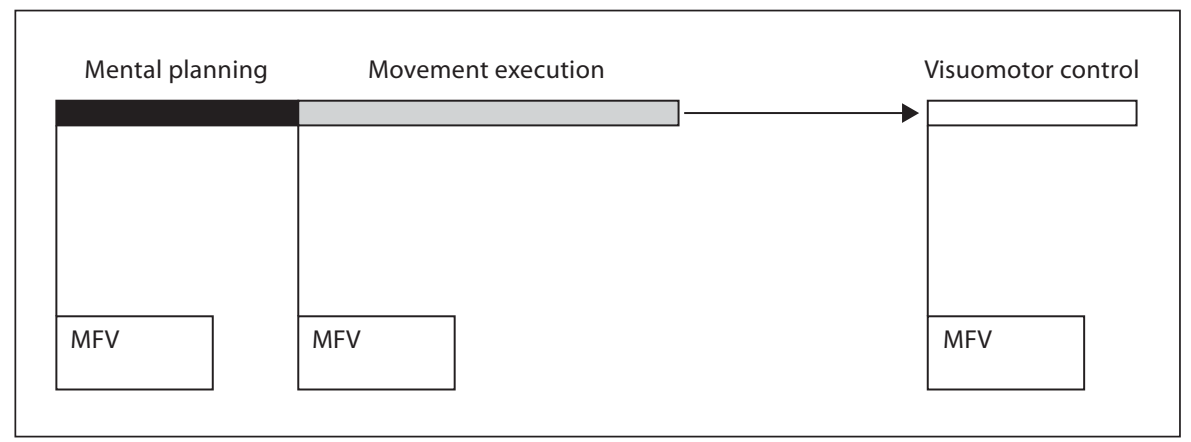

The predetermined number of minimum moves differed from task to task. One block consisted of a 2-, 3-, 4- and 5-move problem with two different trials per problem, respectively (8 problems in total). Each block started with a 2-move problem while the following 3-, 4- and 5-move problems were assessed in random order with a pause of $20 \mathrm{~s}$ between each problem [for details, see 18].

\section{Data Collection}

\section{Performance}

The number of moves above average was taken as a measure of task accuracy, as was the number of perfect solutions. Further, the adjusted planning time [19] and the movement execution time were registered. We differentiated between easy (average of 2- and 3 -move problems) and difficult problems (average of 4- and 5 -move problems) [11] instead of a parametric design.

Mean Cerebral Blood Flow Velocity

Time intervals of interest were gathered around two different time intervals, on the one hand to minimize the amount of missing values and on the other hand to maximize data output. Using a priori knowledge on cerebral hemodynamics during SOC [18, 20], we set the time interval of interest for MFV analysis at $0-2 \mathrm{~s}$ for easy problems and $0-5 \mathrm{~s}$ for difficult ones (planning, movement execution and control).

Offline analysis of MFV comprised the following steps [12]: (a) offline export of the digitized MFV (sampling frequency $100 \mathrm{~Hz}$ ) data to a commercially available spreadsheet program (MS-Excel, Microsoft Corp.); (b) integration of MFV from 100-Hz sampling to $1 \mathrm{~Hz}$, i.e. 1 value/s, and (c) normalization of digitized data with reference to pre- and post-task rest phases (60-second intervals of rest with $30 \mathrm{~s}$ between the first and last $15 \mathrm{~s}$ ). These relative MFV (relative to the resting state) values were then converted to percentage values. All MFV values in this paper are relative MFV. Because of the rapid performance of SOC, $1.20 \%$ of the MFV values were missing, which were replaced by cells calculated by multiple linear regressions [17].

Laterality

In order to examine hemispheric differences during SOC, the laterality index (LI') was assessed as defined elsewhere [adapted from 21]:

$$
\mathrm{LI}^{\prime} \text { = relative } \mathrm{MFV} \text { (right MCA) - relative MFV (left MCA) }
$$

We calculated LI' on a second-wise basis to describe instantaneous changes over time. Significant values in statistical analyses were further examined by the hemispheric advantage given as: laterality = LI' paradigm - LI' control where 'control' is defined as the visuomotor control task of the SOC. Positive values of laterality imply lateralization to the right and negative values imply lateralization to the left. Zero laterality may indicate no change with respect to the control condition or equally bilateral activation. In other words, laterality during SOC as used in statistical analyses is controlled for visuomotor activity.

\section{Statistical Analyses}

The Kolmogorov-Smirnov test was applied to test for normality of distribution $(\mathrm{p}<0.05)$. Data are presented as mean \pm standard deviation.

\section{Performance}

Between-group differences of performance were evaluated using separate repeated measures analyses of variance with gender as a between-subject factor, task difficulty as a within-subject factor (easy and difficult problems) and the respective performance variable (e.g. adjusted planning time) as a dependent measure.

\section{Laterality}

We examined the hemispheric dominance of the entire sample by repeated measures multivariate analyses of variance with condition (planning, movement execution) and time (0, 1 and $2 \mathrm{~s}$ for easy and $0,1,2,3,4$ and $5 \mathrm{~s}$ for difficult problems) as within-subject factors and LI as a dependent measure. Significant effects were followed by univariate analyses where appropriate. Corresponding analyses were also performed using gender as a between-subject factor.

Laterality and Performance

To address the linkage between performance and lateralization for the entire sample and gender-wise, we applied the following analyses:

(a) Between-measures of performance (task accuracy, adjusted planning time and movement execution time) and mean LI during mental planning and movement execution were calculated using Pearson's product moment correlation coefficient. These analyses were carried for behavior and LI of the same phase as well as 'crossover modality' between, for example, performance during mental planning and LI during movement execution. To avoid possible alpha inflation due to multiple testing, we set alpha at a value of 0.01 . The results of second-wise LI and performance are not shown (available upon request). 
Table 1. Performance during easy and difficult problems

\begin{tabular}{|c|c|c|c|c|c|c|}
\hline & $\begin{array}{l}\text { Males } \\
(\mathrm{n}=14)\end{array}$ & $95 \% \mathrm{CI}$ & $\begin{array}{l}\text { Females } \\
(\mathrm{n}=14)\end{array}$ & $95 \% \mathrm{CI}$ & d.f. & F or $\mathrm{z}$ \\
\hline \multicolumn{7}{|l|}{ I. Easy problems } \\
\hline Adjusted planning time, $s$ & $3.88 \pm 2.01$ & $2.72-5.04$ & $3.24 \pm 2.12$ & $2.02-4.47$ & 1,26 & 0.66 \\
\hline Movement execution time, $s$ & $4.04 \pm 1.88$ & $2.95-5.12$ & $4.54 \pm 1.66$ & $3.58-5.49$ & 1,26 & 0.56 \\
\hline Moves above minimum & $0.23 \pm 0.39$ & & $0.11 \pm 0.16$ & & & $1.04^{\mathrm{a}}$ \\
\hline Number of perfect solutions & $3.29 \pm 0.83$ & & $3.64 \pm 0.50$ & & & $1.23^{\mathrm{a}}$ \\
\hline \multicolumn{7}{|l|}{ II. Difficult problems } \\
\hline Adjusted planning time, $\mathrm{s}$ & $12.57 \pm 8.30$ & $7.78-17.36$ & $8.90 \pm 6.31$ & $5.25-12.54$ & 1,26 & 1.74 \\
\hline Movement execution time, s & $20.95 \pm 21.69$ & $8.43-33.48$ & $32.00 \pm 26.80$ & $16.52-47.47$ & 1,26 & 1.44 \\
\hline Moves above minimum & $1.34 \pm 1.42$ & $0.52-2.16$ & $1.84 \pm 1.53$ & $0.95-2.72$ & 1,26 & 0.80 \\
\hline Number of perfect solutions & $2.79 \pm 1.12$ & $2.14-3.43$ & $2.14 \pm 1.17$ & $1.47-2.82$ & 1,26 & 2.21 \\
\hline
\end{tabular}

Values are means $\pm \mathrm{SD}$. All $\mathrm{p}$ values $\geq 0.2 .{ }^{\mathrm{a}} \mathrm{Z}$ value of Mann-Whitney $\mathrm{U}$ test.

(b) In an attempt to hemodynamically characterize optimal performers of difficult problems from the rest of the sample, second-wise change scores of LI were calculated (termed LI change) and examined by an independent sample t test between groups. Second-wise change scores proved to be a fruitful strategy to investigate brain behavior relationships in other studies [10, 18].

Alpha was set at 0.05 if not otherwise stated.

\section{Results}

\section{Performance}

A highly significant effect of problem difficulty was found for all performance variables $(p<0.001)$, but no main effect of gender $(p>0.18)$ or a significant gender by problem difficulty interaction was found $(p>0.25)$. There were no significant differences of individual test scores between men and women ( $p>0.20)$ (table 1). Seven subjects solved difficult problems in the minimum number of moves (i.e. optimal performance).

\section{Laterality}

The time course for the entire sample and gender-wise is given in figures 2 and 3, respectively. LI outlier data of 1 female subject was discarded because values were more than 2 standard deviations above the mean values.

\section{Easy Problems}

(a) Entire sample: there was a main effect of time (Wilks' lambda $=0.74, \mathrm{~F}(2,25)=4.44, \mathrm{p}=0.022)$ indicating some temporal variation of hemispheric dominance during both mental planning and movement execution (fig. 2a). (b) Gender: there were significant condition by gender (Wilks' lambda $=0.69 \mathrm{~F}(1,25)=11.13, \mathrm{p}=0.003)$, time by gender (Wilks' lambda $=0.71, \mathrm{~F}(2,24)=4.63, \mathrm{p}=0.017$ ) and condition by time by gender interactions (Wilks' lambda $=0.70, F(2,24)=5.15, p=0.014)$. In females, there was a significant shift to left hemispheric lateralization during movement execution (fig. 3a, b) compared to plan$\operatorname{ning}(-1.28 \pm 2.35$ vs. $0.10 \pm 2.03, \mathrm{t}(12)=3.06, \mathrm{p}=0.010)$, which was not true for males $(0.05 \pm 2.65$ vs. $0.40 \pm 2.86$, $\mathrm{t}(13)=1.28, \mathrm{p}=0.22)$. We found a temporal variation of LI during movement execution in females $(\mathrm{F}(2,11)=6.91$, $\mathrm{p}=0.011$ ); post hoc analyses with pair-wise analyses, using a Bonferroni correction for multiple comparisons $(\mathrm{p}<0.05)$, showed a left hemispheric dominance at $1 \mathrm{~s}$ (fig. 3b).

\section{Difficult Problems}

(a) Entire sample: there was a main effect of condition (Wilks' lambda $=0.79, \mathrm{~F}(1,26)=6.86, \mathrm{p}=0.015$ ), and time (Wilks' lambda $=0.60, \mathrm{~F}(5,22)=2.97, \mathrm{p}=0.034)$ and $\mathrm{a}$ highly significant condition by time interaction (Wilks' lambda $=0.39, \mathrm{~F}(5,22)=7.01, \mathrm{p}<0.001)$. Post hoc analysis using pair-wise analysis, with a Bonferroni correction for multiple comparisons $(\mathrm{p}<0.05)$, yielded increased LI to the right during mental planning compared to movement execution $(0.87 \pm 2.46$ vs. $-0.20 \pm 2.95, \mathrm{t}(26)=$ $0.015)$ (fig. 2b). There was a significant lateralization to the right $(\mathrm{F}(5,22)=4.25, \mathrm{p}=0.007)$ compared to the start (fig. 2b).

(b) Gender: we found a significant time by gender interaction (Wilks' lambda $=0.61, \mathrm{~F}(5,21)=2.68, \mathrm{p}=0.050$ ) (fig. $3 \mathrm{c}, \mathrm{d}$ ). In males, there was LI to the right during late 
Fig. 2. a, b Lateralization during easy and difficult SOC versions $(\mathrm{n}=27)$. ${ }^{*} \mathrm{p}<0.05$, ** $\mathrm{p}<0.01$ (compared to lateralization at $0 \mathrm{~s})$. a Planning and movement execution, easy problems. b Planning and movement execution, difficult problems.
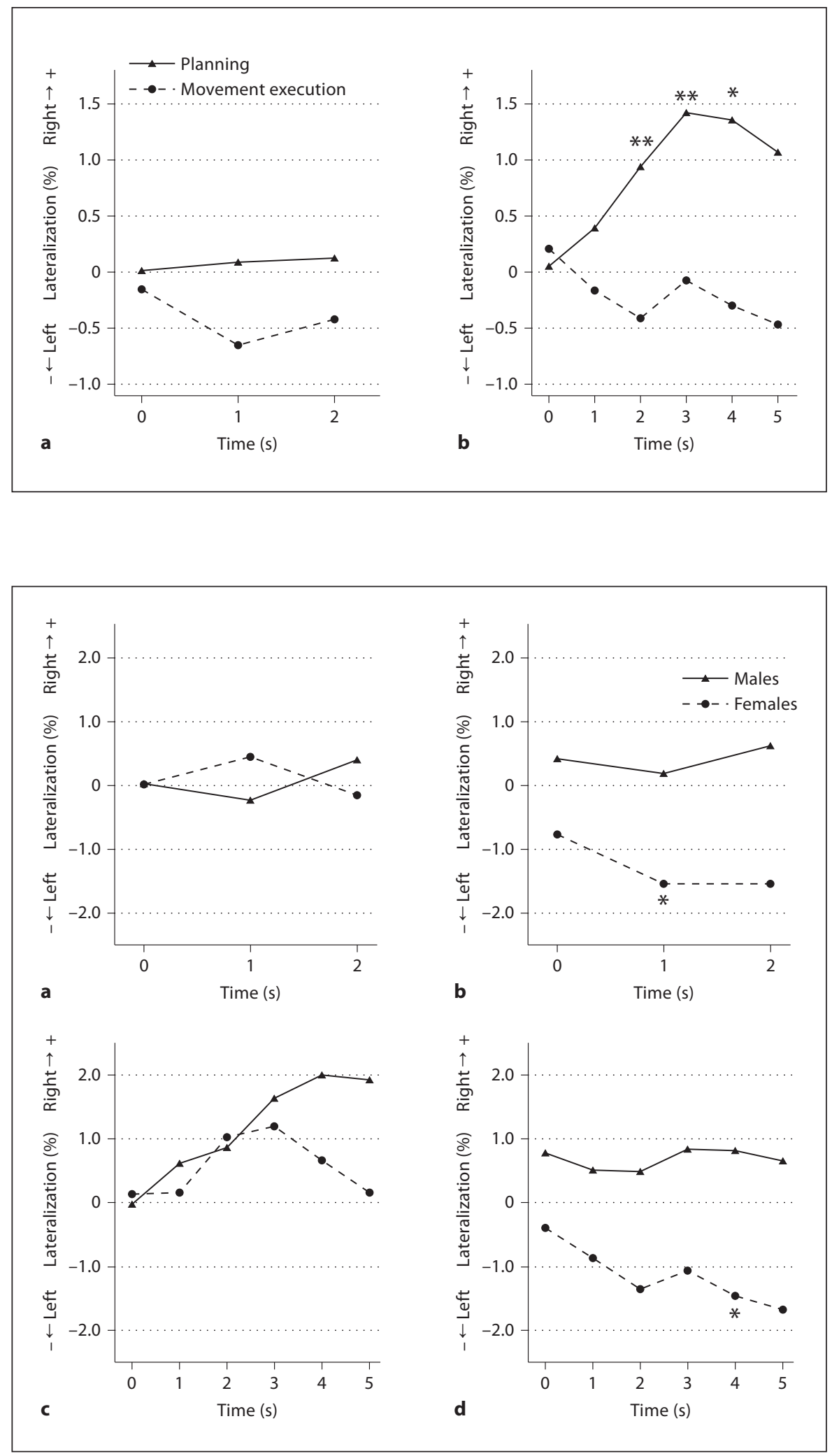

Fig. 3. a-d Gender-wise lateralization during easy and difficult SOC versions $(n=14$ for males and $n=13$ for females, respectively). ${ }^{*} \mathrm{p}<0.05$ (compared to lateralization at $0 \mathrm{~s}$ ). a Planning, easy problems. b Movement execution, easy problems. c Planning, difficult problems. d Movement execution, difficult problems. 


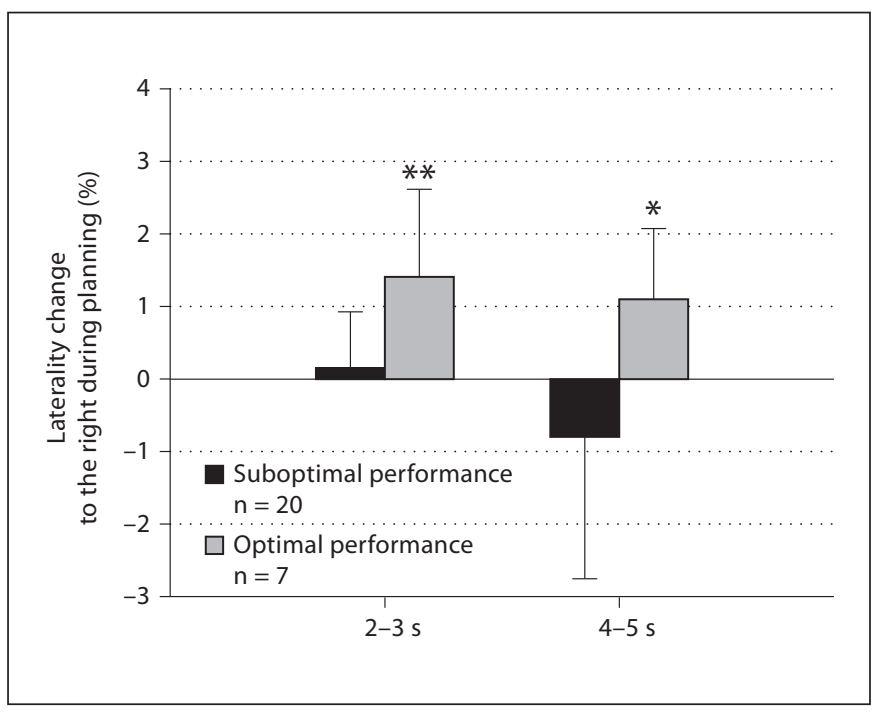

Fig. 4. Optimal performance and lateralization change (intervals of $2-3 \mathrm{~s}$ and $4-5 \mathrm{~s}$ after the start) during difficult mental planning. ${ }^{*} \mathrm{p}=0.022,{ }^{* *} \mathrm{p}=0.004$.

planning (online suppl. table 1, for all online suppl. material, see www.karger.com/doi/10.1159/000339562). During movement execution, females showed left hemispheric lateralization $(\mathrm{F}(5,8)=3.87, \mathrm{p}=0.044)$ at the later phase (fig. 3d). On a trend level, there were similar interactions to those observed during easy problems (data not shown).

The planning of difficult problems led to a right hemispheric dominance, especially during the late phase. Females were left hemisphere dominant during movement execution, whereas males showed a bilateral pattern. We detected temporal variations of hemispheric dominance.

\section{Laterality and Performance}

Easy Problems

No significant associations were found that survived the significance threshold.

\section{Difficult Problems}

(a) Entire sample: LI to the left during movement execution correlated with shorter planning $(\mathrm{r}=0.59, \mathrm{p}=$ 0.001 ). When investigating potential LI differences between those participants with best performance $(n=7)$ in terms of perfect solutions (i.e. 4 difficult problems) and the rest of the sample $(n=20)$, the best performers showed an increasing LI change to the right between $2-3 \mathrm{~s}$ and 4-5 s (fig. 4).

(b) Gender: as for the entire sample, female participants yielded a linkage between increased LI to the left during movement execution and shorter planning times $(r=0.80, p=0.001)$. Further, there was an association between LI to the left during mental planning and shorter planning times in this group $(\mathrm{r}=0.75, \mathrm{p}=0.003)$.

An increase in right hemispheric dominance during difficult mental planning was associated with optimal solutions. Left hemispheric lateralization during difficult movement execution was correlated with shorter planning times, especially in females.

\section{Discussion}

We observed diverse patterns of hemispheric lateralization during planning, and relevant cofounders were task difficulty, task phase and gender. These patterns proved to be behaviorally relevant in a multifaceted way. The main results can be summarized as follows: first, difficult mental planning provoked lateralization to the right hemisphere for the late phase that was not found during movement execution. Here, it is of relevance that right hand/finger activity was properly controlled for. Second, females used a switching strategy of lateralization during easy problems, i.e. a bilateral pattern during planning and a shift to the left during movement execution. Similar patterns were found for difficult problems. Third, hemispheric lateralization played a distinct role when it came to task performance: subjects with optimal performance had an increasing lateralization change to the right during difficult planning problems.

\section{Laterality}

Mental planning without motor action provoked a shift to the right hemisphere, a feature that was exclusively observed during difficult problems. It took $2 \mathrm{~s}$ until the shift became apparent. This feature was not present during easy problems, and statistical analyses comparing both difficult levels during 0-2 s showed a significant difference between easy and difficult problems when considering condition and time (data not shown). These results support evidence that easy and difficult planning problems elicit different cerebral hemodynamic patterns [11]. Unterrainer et al. [3] emphasized the crucial role of the right DLPFC in higher level problem solving such as planning, but also the parietal regions such as the inferior parietal cortex. The right DLPFC lies within the territory of the right MCA; therefore, we conclude that results of this fTCD study, namely LI to the right during planning, fit nicely with those presented by Unterrainer et al. [3]. Woolley et al. [22] observed more activation in 
the right than in the left hemisphere when online visuospatial information was used to control and correct ongoing movements. Although SOC is not a pure motor task, planning ahead may be regarded as preparation to orderly control appropriate movements. Serrien et al. [23] suggested, within the context of motor function, that right hemispheric activation requires a strong external stimulus for the selection of a distinct motor representation from various options, or a selection mechanism that involves predominantly exploratory processing of novel situations. For the sake of completion, the right hemisphere also plays an important role in social information processing [24]. Newman et al. [25] proposed that the right DLPFC is involved in strategy formation and its activation depends on the level of difficulty. With respect to the 'late' right hemispheric shift during difficult planning, the cerebral hemodynamic response takes about $2 \mathrm{~s}$ to evolve, is peaking at $3 \mathrm{~s}$, and is therefore not a constant phenomenon. Difficult SOC problems include complex and novel external stimuli with spatial planning features. This supports the notion of a dynamic pattern of laterality. Serrien et al. [23] emphasized the role of hemispheric specialization within the context of motor action. They proposed a dynamic disposition of lateralized motor function characterized by hemispheric and interhemispheric dynamics across different time scales. The left hemisphere has been linked to higher-order aspects of action that are related to movement complexity [23]. Furthermore, it has been proposed that left hemispheric activity is of importance for temporal processing of sequential movements [26]. It is least surprising that functions both of the right and left hemisphere are required to adequately solve a complex planning paradigm such as SOC. This is true for the movement execution phase, when a prepared plan has to be carried out (temporal processing of sequential movements), online planning is required to correct false plans [27] and verbal and visuospatial information abilities are used. As stated in Frauenfelder et al. [11], both left and right hemispheres are necessary to successfully solve SOC. Obviously, the planning of difficult spatial problems predominantly requires right hemispheric activity, although in a dynamic and diverse manner. It has to be presumed, based on the available evidence, that the right DLPFC is more involved than the left in difficult mental planning.

\section{Laterality and Gender}

Common and separate features between males and females were observed during SOC. Both groups showed LI to the right, at least at trend level, during mental plan- ning. During the movement execution phase, females showed LI to the left whereas males yielded a bilateral pattern. These patterns did not evolve immediately, but took 1-4 s (see fig. 3b, d). In other words, females shifted from a slightly right hemispheric LI pattern during mental planning to the left during movement execution, and males were bilateral during that phase. These results are in line with another study [28] with a right hemispheric dominance of males during a general intelligence task and LI to the left for females. The study suggested that right hemispheric dominance in males enhances visuospatial problem solving. In contrast, females used a switching strategy of lateralization, from initially a rather right or bilateral (mental planning) to a left hemispheric dominance (movement execution). Similar results have been found more subtly in a very recent fTCD study using Trail Making, Part B [12]. In other words, mental planning showed about the same LI for both males and females. However, the execution of a plan provoked diverse LI patterns, implying distinct cognitive styles when solving spatial planning paradigms. Also, an fMRI study on separate phases of a planning task found gender differences during movement execution but not during mental planning [3]. However, these authors observed a slight performance decrement during movement execution in females compared to males. Our results did not show significant performance differences during that phase, but a careful inspection yielded somewhat longer movement execution times and moves above minimum for females (table 1).

\section{Laterality and Performance}

The most important result in this study was that optimal performers showed a significant LI change to the right during difficult mental planning. Laterality itself, either in a static way as mean value or second-wise, was not associated with best or suboptimal performance in terms of perfect solutions. Right hemispheric dominance enhances spatial problem solving, and dynamic changes of laterality have to be considered [23]. Other fTCD studies have shown promising results of brain behavior relationships when using second-wise change measures of MFV or very short MFV intervals $[9,10,18]$.

In this study, there have been further associations between performance and mean laterality. Left hemispheric lateralization during movement execution was associated with shorter planning times. This was true for the entire sample and for females. In addition, this latter group yielded LI to the left during mental planning with 
shorter planning times. In other words, increased planning speed and left lateralization were associated. However, this was not true for speed during movement execution or other performance variables. We hypothesize that LI to the left represents an emphasis on temporal processing of sequential movements [26]. Since there was no significant association with other performance variables during difficult planning conditions, we conclude that LI to the left is not necessarily an advantage when solving a visuospatial planning paradigm such as SOC. It appears more plausible that both left and right hemispheres are necessary to achieve an optimal strategy, or, when planning without motor activity is required, a shift to the right hemisphere.

In other words, laterality or its dynamic equivalent plays a role in speed and optimal performance during SOC. Unterrainer et al. [3] described a linear relationship between the right DLPFC and individual planning performance. Their study concluded that the right DLPFC plays a strong role in the generation of plans for complex visuospatial tasks.

\section{Study Limitation}

The group of optimal performers was rather small, and a larger sample size should confirm the results of our study.

\section{Conclusions}

This study on lateralization during planning found patterns of hemispheric dominance that correspond well with the available literature. Difficult mental planning provoked lateralization to the right. This finding suggests a marked activation of the right lateral hemisphere which itself harbors structures essential for the selection of a distinct motor representation such as the right DLPFC and also the right parietal regions. We observed, exclusively in females, a left hemispheric dominance during movement execution implying an emphasis on strategic aspects and, hence, a diverse cognitive style when solving planning tasks. Most importantly, optimal performance was associated with a rapid change of laterality to the right during mental planning compared to suboptimal performance. This latter result emphasizes the importance of lateralization and its dynamics for best performance of a complex cognitive task. Not only hemispheric laterality, but also its rapid shift may be used as a marker of performance enhancement. Future studies should examine the role of laterality and its dynamics in disorders with abnormal lateralization patterns such as schizophrenia.

\section{Acknowledgement}

This study has been supported by the Hartmann Müller Foundation for Medical Research, Zurich, Switzerland.

\section{References}

1 Fuster JM: The Prefrontal Cortex, ed 4. Amsterdam, Elsevier, 2008.

2 Shallice T: Specific impairments of planning. Phil Trans R Soc Lond B 1982;298:199-209.

3 Unterrainer JM, Ruff CC, Rahm B, Kaller CP, Spreer J, Schwarzwald R, Halsband U: The influence of sex differences and individual task performance on brain activation during planning. Neuroimage 2005;24:586590 .

4 Boghi A, Rasetti R, Avidano F, Manzone C, Orsi L, D'Agata F, Caroppo P, Bergui M, Rocca P, Pulvirenti L, Bradac GB, Bogetto F, Mutani R, Mortara P: The effect of gender on planning: an fMRI study using the Tower of London task. Neuroimage 2006;33:9991010.

5 Jordan K, Würstenberg T, Heinze HJ, Peters $\mathrm{M}$, Jäncke L: Women and men exhibit different cortical activation patterns during mental rotation tasks. Neuropsychologia 2002; 40:2397-2408.
6 Neubauer AC, Grabner RH, Fink A, Neuper $\mathrm{C}$ : Intelligence and neural efficiency: further evidence of the influence of task content and sex on the brain-IQ relationship. Cog Brain Res 2005;25:217-225.

7 Bell EC, Willson MC, Wilman AH, Dave S, Silverstone PH: Males and females differ in brain activation during cognitive tasks. Neuroimage 2006;30:529-538.

8 Duschek S, Schandry R: Functional transcranial Doppler sonography as a tool in psychophysiological research. Psychophysiology 2003;40:436-454.

9 Duschek S, Schuepbach D, Schandry R: Time-locked association between rapid cerebral blood flow modulation and attentional performance. Clin Neurophysiol 2008;119: 1292-1299.
10 Schuepbach D, Huizinga M, Duschek S, Grimm S, Boeker H, Hell D: Rapid cerebral hemodynamic modulation during set shifting: evidence of time-locked associations with cognitive control in females. Brain Cog 2009;71:313-319.

11 Frauenfelder BA, Schuepbach D, Baumgartner RW, Hell D. Specific alterations of cerebral hemodynamics during a planning task: a transcranial Doppler sonography study. Neuroimage 2004;22:1223-1230.

12 Misteli M, Duschek S, Richter A, Grimm S, Rezk M, Kraehenmann R, Boeker H, Seifritz E, Schuepbach D: Gender characteristics of cerebral hemodynamics during complex cognitive functioning. Brain Cog 2011;76: 123-130.

13 Salmaso D, Longoni AM: Problems in the assessment of hand preference. Cortex 1985;21: 533-549. 
14 Willie CK, Colino FL, Bailey DM, Tzeng YC, Binsted G, Jones LW, Haykowsky MJ, Bellapart J, Ogoh S, Smith KJ, Smirl JD, Day TA, Lucas SJE, Eller LK, Ainslie PN: Utility of transcranial Doppler ultrasound for the integrative assessment of cerebrovascular function. J. Neurosci Methods 2011;196:221237.

15 Moehring MA, Spencer MP: Power M-mode Doppler (PMD) for observing cerebral blood flow and tracking emboli. Ultrasound Med Biol 2002;28:49-57.

16 Serrador JM, Picot PA, Rutt BK, Shoemaker JK, Bondar RL: MRI measures of middle cerebral artery diameter in conscious humans during simulated orthostasis. Stroke 2000; 31:1672-1678.

17 Feldmann D, Schuepbach D, von Rickenbach B, Theodoridou A, Hell D: Association between two distinct executive tasks in schizophrenia: a functional transcranial Doppler sonography study. BMC Psychiatry 2006;6: 25.
18 Schuepbach D, Boeker H, Duschek S, Hell D Rapid cerebral hemodynamic modulation during mental planning and movement execution: evidence of time-locked relationship with complex behavior. Clin Neurophysiol 2007; 118:2254-2262.

19 Owen AM, Downes JJ, Sahakian BJ, Polkey CE, Robbins TW: Planning and spatial working memory following frontal lobe lesions in man. Neuropsychologia 1990;28: 1021-1034.

20 Schuepbach D, Weber S, Kawohl W, Hell D: Impaired rapid modulation of cerebral hemodynamics during a planning task in schizophrenia. Clin Neurophysiol 2007;118: 1449-1459.

21 Knecht S, Deppe E-B, Ringelstein, M, Wirtz M, Lohmann H, Dräger B, Huber T, Henningsen $\mathrm{H}$ : Reproducibility of functional transcranial Doppler sonography in determining hemispheric language lateralization. Stroke 1998;29:1155-1159.

22 Woolley DG, Wenderoth N, Heuninckx S, Zhang X, Callaert D, Swinnen SP: Visual guidance modulates hemispheric asymmetries during an interlimb coordination task. Neuroimage 2010;50,1566-1577.
23 Serrien DJ, Ivry RB, Swinnen SP: Dynamics of hemispheric specialization and integration in the context of motor control. Nat Rev Neurosci 2006; 7:160-166.

24 Semrud-Clikeman M, Fine JG, Zhu DC: The role of the right hemisphere for processing of social interactions in normal adults using functional magnetic resonance imaging. Neuropsychobiology 2011;64:47-51.

25 Newman SD, Carpenter PA, Varma S, Just MA: Frontal and parietal participation in problem solving in the Tower of London: fMRI and computational modelling of planning and high-level perception. Neuropsychologia 2003;41:1668-1682.

26 Agnew JA, Zeffiro TA, Eden GF: Left hemispheric specialization for the control of voluntary movement rate. Neuroimage 2004; 22:289-303.

27 Ward G, Allport A: Planning and problemsolving using the five-disc Tower of London task. Q J Exp Psychol A 1997;50:49-78.

28 Njemanze PC: Cerebral lateralization and general intelligence: gender differences in a transcranial Doppler study. Brain Lang 2005;92:234-239. 